\title{
Role of the first mitosis in the remodeling of the parental genomes in mouse embryos
}

\author{
Hong Lin LIU ${ }^{1,2}$, Kentaro T. HARA ${ }^{1}$, Fugaku AOKI ${ }^{1, *}$ \\ ${ }^{1}$ Department of Integrated Biosciences, Graduate School of Frontier Sciences, University of Tokyo, Kashiwa, Chiba $277-$ \\ 8571, Japan \\ ${ }^{2}$ College of Animal Science and Technology, Nanjing Agricultural University, Nanjing 210095, China.
}

\begin{abstract}
Although male and female pronuclei reside in the same zygotic cytoplasm, they differ in many respects, such as volume and transcriptional activity. The aim of this study is to investigate whether these differences are lost during the first mitosis. For this purpose, a new method was developed to inhibit the mixing of two parental chromosomes during mitosis, thus to induce the formation of two nuclei after they exit from the mitotic phase. In this method, one-cell embryos are arrested at metaphase by treatment with nocodazole, and whn exitting from the mitotic phase, two nuclei were formed in a single karyocyte following treatment with 6-dimethylaminopurine (6-DMAP). These embryos were designated as post-mitotic embryos (PM-embryos), in which the two nuclei were derived from the male and female genomes. We found that in the control one-cell embryos that had not been treated with the reagents, the volume of the male pronucleus was about 1.65-fold greater than that of the female pronucleus, whereas the volumes of the two nuclei in the PM-embryos were similar (volume ratio of 1.01). Although a two-fold difference in transcriptional activity was detected between the male and female pronuclei in the control embryos, no difference in transcriptional activity was detected between the two nuclei of PM-embryos. The ratio of transcriptional activity in the nucleus derived from the paternal genome to that from the maternal genome was 1.02 , for which no significant difference was detected by the $\chi^{2}$ fitness test. Therefore, the volumes and transcriptional activities of the male and female nuclei were approximately equal in PM-embryos, which suggests that the asymmetries of pronuclear volume and transcriptional activity between male and female genomes are somehow losted during the first mitosis.
\end{abstract}

Keywords: mouse zygote, parental genomes, nuclear volume, transcriptional activity.

\section{INTRODUCTION}

Although the major burst of gene expression occurs during the two-cell stage in mice, recent evidence suggest that the one-cell-stage embryo is transcriptional active, and the male pronucleus (PN) supports a significantly higher level of transcription than the female PN [1]. This discrepancy may be attributed to the protamine-histone exchange that occurs in the male PN soon after fertilization. Compared to the female PN, the male PN has a larger volume, higher concentrations of transcription factors, higher levels of histone acetylation [2-4], undergoes extensive DNA demethylation [5], and is more sensitive to

*Correspondence: Fugaku AOKI

Tel: +81-4-7136-5424, Fax: +81-4-7136-3698,

E-mail: aokif@k.u-tokyo.ac.jp
DNase I digestion [6]. Thus, the male and female genomes are asymmetric in many aspects during the one-cell stage. However, it has not been clarified whether these differences remain after cleavage to the two-cell stage, when the chromosomes of two parental origins are unified in the nucleus.

The transcriptional programs of the eukaryotic cell are closely correlated with the cell cycle, for example, sequencespecific transcription factors are displaced from mitotic chromatin and then transcription ceases [7]. Therefore, we hypothesized that the asymmetry between the two parental genomes could be lost during the first mitosis. However, it used to be difficult to address this possibility, since it's very hard to inhibit the mingling of parental genomes during the first mitosis. The parental genomes are mixed when the zygote enters into the metaphase of the first cell cycle, and are subsequently distributed equally to 
the two nuclei when exiting the interphase of the two-cell stage. In this study, a new method was developed, which could specifically inhibit the mixing of parental genomes during the first mitosis while permitting chromatin condensation and nuclei formation. Using this method, we demonstrated that the asymmetries of pronuclear volume and transcriptional activity between the male and female genomes was somewhat lost during the first mitosis.

\section{MATERIALS AND METHODS}

\section{In vitro fertilization and culturing of embryos}

Female mice at 21-23 d (ddY-SLC; SLC, Shizuoka, Japan) were superovulated with $5 \mathrm{IU}$ of pregnant mare serum gonadotropin (Sankyo Co., Ltd., Tokyo, Japan) and treated $48 \mathrm{~h}$ later with $5 \mathrm{IU}$ of human chorionic gonadotropin (hCG; Sankyo). Oocytes were collected in Whitten's medium [8] from the ampullae of the oviduct 15-16 h after hCG injection. Sperm were obtained from the cauda epididymis of the mature male ICR mouse and added into Whitten's medium. Oocytes were inseminated with capacitated sperm that had been initially incubated for $2 \mathrm{~h}$ at $37^{\circ} \mathrm{C}$. Four hours after insemination, the embryos were washed with glucose-free CZB medium [9] and cultured in a humidified incubator of $5 \% \mathrm{CO}_{2} / 95 \%$ air at $37^{\circ} \mathrm{C}$. To inhibit the first round of DNA replication, embryos were exposed to $3 \mu \mathrm{g} / \mathrm{ml}$ aphidicolin before the zygotes entered the $\mathrm{S}$ phase.

\section{Treatment of nocodazole and 6-dimethylaminopurine (6- DMAP) to produce PM-embryos}

A new method was designed to produce embryos in which the parental genomes separate form the nuclei in a single karyocyte after exiting from the first mitosis. These embryos were designated as post-mitotic embryos (PM-embryos). Embryos with two pronuclei were collected $12 \mathrm{~h}$ after insemination and transferred into CZB medium containing $0.5 \mu \mathrm{g} / \mathrm{ml}$ nocodazole, in order to arrest the cell cycle at metaphase. Four hours later, $8 \mathrm{mM}$ 6-DMAP was added to the medium. Nuclei were formed after being cultured for $3 \mathrm{~h}$ in the presence of nocodazole and 6-DMAP. The embryos were then washed and cultured in CZB medium without treatment for $5 \mathrm{~h}$, and the volumes and transcriptional activities of the nuclei were examined.

\section{Measurement of nuclear volume}

Embryos were fixed in 3.7\% paraformaldehyde and stained with $3 \mu \mathrm{g} / \mathrm{ml} \mathrm{4,6-diamidino-2-phenylindole} \mathrm{(DAPI)} \mathrm{for} 20 \mathrm{~min}$. The cells were mounted on a slide in the anti-bleaching solution Vectashield (Vector Laboratories, Burlingame, CA, USA). Measurements of pronucleus/nucleus volume were carried out using a Leica TCS SP2 laserscanning confocal microscope. The pronucleus/nucleus volumes of the embryos were obtained by multiplying the maximum pronucleus/ nucleus sections by the thickness of pronucleus/nucleus along the $z$ axis. For each embryo, the ratio of the volume of the male pronucleus to that of the female pronucleus was calculated.

\section{Immunochemistry stainning}

Embryos were washed in PBS containing $3 \mathrm{mg} / \mathrm{ml}$ polyvinylpyrrolidone (PBS/PVP), fixed for $1 \mathrm{~h}$ in $3.7 \%$ paraformaldehyde in PBS, and permeabilized with $0.5 \%$ Triton X-100 in PBS for 20 min at room temperature. Then the cells were incubated for $1 \mathrm{~h}$ with a 1:200 dilution of the rabbit polyclonal antibody which specially recognizes dimethyl-lysine 9 on histone H3 (MeH3K9) (Upstate Biotechnology,
Lake Placid, NY, USA), and then for $1 \mathrm{~h}$ with a secondary FITCconjugated antibody (Jackson ImmunoResearch, West Grove, PA, USA). DAPI was used to mark the DNA of the cells. The cells were mounted on a glass slide in Vectashield and the fluorescence was detected using a Leica TCS SP2 laser-scanning confocal microscope.

\section{In vitro transcription assay}

The transcriptional activities of the embryos were determined by measuring the incorporation of 5-bromouridine-5-triphosphate (BrUTP; Sigma Chemical Co., St. Louis, MO, USA) into plasma membrane-permeabilized embryos [1]. Briefly, the plasma membranes of the embryos were permeabilized by treatment with $0.05 \%$ Triton X-100 for 1-2 min. Permeabilized embryos were subjected to in vitro transcription reactions in which UTP was replaced by BrUTP in the reaction mixture. After incubation for $15 \mathrm{~min}$ at $33^{\circ} \mathrm{C}$, the embryos were fixed overnight with $3.7 \%$ paraformaldehyde. Permeabilization of the nuclear membranes was performed on fixed oocytes with $0.2 \%$ Triton X-100 for 3 min. Incorporated BrU was detected by immunostaining with an anti-BrdU antibody, together with a Cy3-conjugated anti-mouse IgG secondary antibody (Jackson ImmunoResearch, PA, USA). In order to identify the male and female nuclei in the PM-embryos, the samples were counterstained with the anti-MeH3K9 antibody. Fluorescence was detected using the Leica TCS SP2 laser-scanning confocal microscope, and the signal intensity was quantified using NIH Image software. The pixel value/ unit area was measured for the nucleus, and the average value of the two different regions of the cytoplasm was subtracted as background. This value was multiplied by the nuclear area to yield the total amount of fluorescence in the nucleus. Embryos were flattened to an extent such that the area could reflect the volume after permeabilization of the plasma membranes with $0.05 \%$ Triton X100. For each embryo, the ratio of male to female pronucleus/nucleus transcriptional activity was calculated.

\section{RESULTS}

\section{Production of PM-embryos by treatment with nocodazole and 6-DMAP}

Male and female pronuclei began to form at $4 \mathrm{~h}$ after insemination (Fig. 1A), and the parental genomes combined and arrayed at the same equator when the embryos entered the $\mathrm{M}$ phase at $16 \mathrm{~h}$ after insemination (Fig. 1B). Initially, the embryos were arrested at metaphase by treatment with nocodazole, which is an inhibitor of microtubule organization. In these embryos, unification of male and female chromosomes was prevented, and these chromosomes were separated into two masses (Fig. 1C). Next, 6-DMAP was added to induce nuclear membrane formation in the nocodazole-treated embryos. We found that the embryos that had been arrested at metaphase by nocodazole formed two nuclei in the presence of 6-DMAP (Fig. 1D). In these nuclei, the chromosomes were decondensed. Moreover, we found that the formation of nuclei in the nocodazole-treated embryos depended on the concentration of 6-DMAP (Fig. 2). Although $2 \mathrm{mM}$ 6-DMAP did not induce any nuclei formation (0/27), $4 \mathrm{mM}$ and $8 \mathrm{mM}$ doses of 6-DMAP induced the formation of nuclei in $46.8 \%$ 

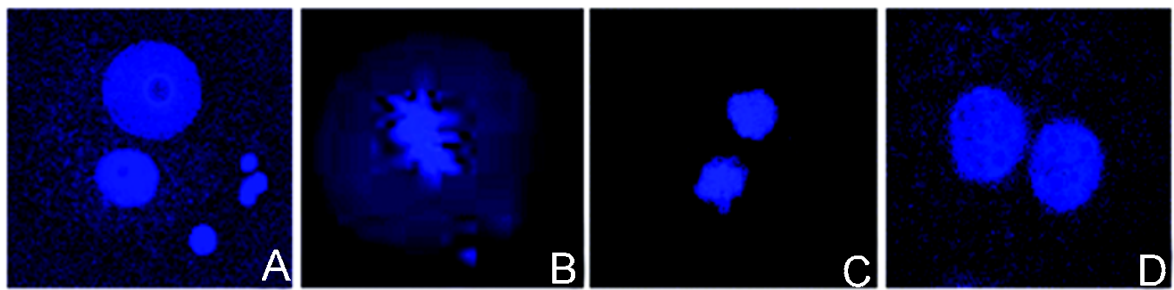

Fig. 1 Confocal microscopy of one-cell embryos stained with DAPI. PM-embryos were produced by treatment with nocodazole and 6-DMAP. Embryos with two pronuclei were culled $12 \mathrm{~h}$ after insemination and transferred into CZB medium that contained $0.5 \mu \mathrm{g} / \mathrm{ml}$ nocodazole, to arrest the cell cycle at metaphase. Four hours later, $8 \mathrm{mM}$ 6-DMAP was added to the medium. Nuclei were formed after culturing for $3 \mathrm{~h}$ in the presence of nocodazole and 6-DMAP. (A) One-cell embryo $6 \mathrm{~h}$ after insemination. (B) One-cell embryo $16 \mathrm{~h}$ after insemination (at M phase). (C) One-cell embryo arrested at the M phase by nocodazole treatment. (D) A PM-embryo.

(22/47) and 97.4\% (76/78) of embryos, respectively.

To confirm that the nuclei in the PM-embryos were derived from maternal and paternal genomes, the H3K9 methylation patterns of the PM-embryos were examined. It has been reported that while H3K9 is methylated asymmetrically in the genomes of maternal and paternal origin in one-cell embryos, only the female genome is highly methylated on $\mathrm{H} 3 \mathrm{~K} 9[10,11]$, and that this asymmetry is maintained after the cleavage into the two-cell stage [12]. In the present study, we confirmed that the maternal genome showed a high level of H3K9 methylation whereas the paternal genome did not in one-cell embryos (Fig. 3A). Similar methylation pattern was observed in the two chromosome masses separated in the metaphase-arrested embryos following nocodazole treatment (Fig. 3B), as well as in the two nuclei formed by subsequent treatment with 6-DMAP (Fig. 3C). These results demonstrate that maternal and paternal genomes have passed through the $\mathrm{M}$ phase and entered interphase reside separately in the two nuclei.

\section{Disappearance of the nuclear volume asymmetry be- tween the two nuclei in the PM-embryo}

In one-cell mouse embryos, the volume of the male pronucleus was larger than that of the female pronucleus: 1.69 -fold at the G1 phase ( $6 \mathrm{~h}$ after insemination) and 1.64-fold at the G2 phase (12 h after insemination) (Fig. 4). This asymmetry of pronucleus volume was maintained up to $24 \mathrm{~h}$ after insemination when the embryos were exposed to the treatment of aphidicolin to inhibit DNA replication. The volume ratio of male/female pronuclei was 1.64 for the aphidicolin-treated embryos. However, the pronucleus volume asymmetry disappeared in the PM-embryos (Fig. 4). As we could not distinguish the nuclei as being derived from the paternal or maternal genome, we calculated the

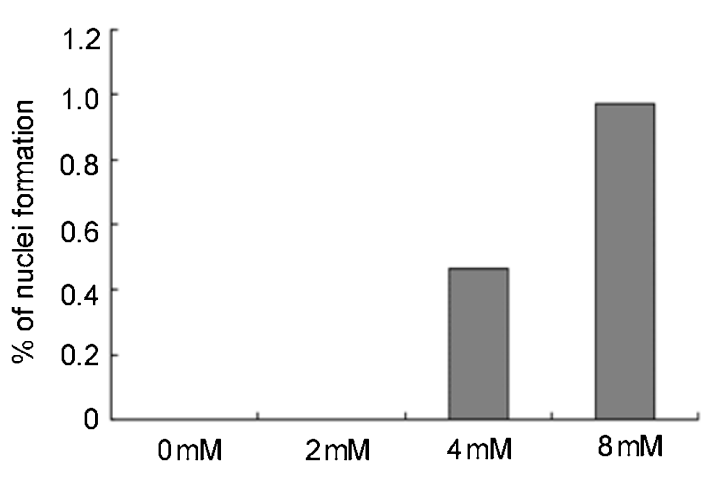

Fig. 2 Effect of 6-DMAP on the formation of nuclei. The percentages of nuclei formation in the embryos treated with various concentration of 6-DMAP are shown. The formation of nuclei was examined $3 \mathrm{~h}$ after 6-DMAP treatment. The numbers of embryos analyzed in the control group (0 mM), 6-DMAP-treated groups ( $2 \mathrm{mM}, 4 \mathrm{mM}$, and $8 \mathrm{mM}$ ) were $50,27,47$, and 78 , respectively.
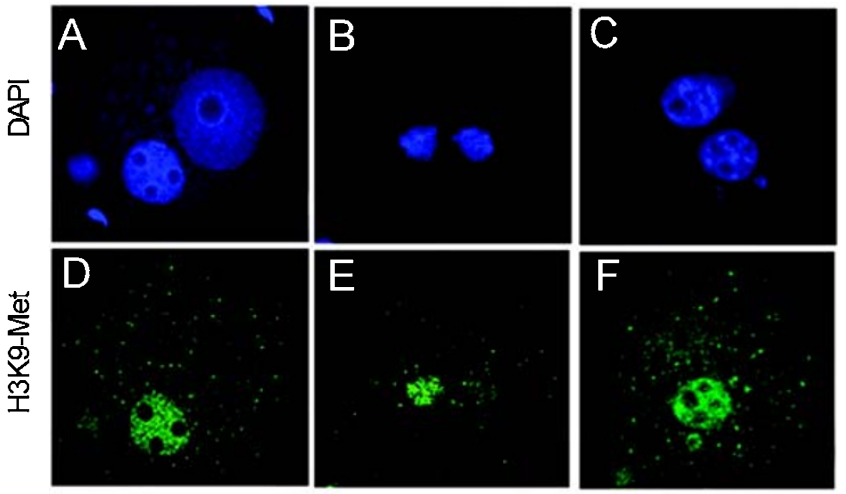

Fig. 3 Methylation of lysine 9 on histone H3 (H3/K9-Met) in onecell embryos. Embryos were immunostained and the images were taken by a laser-scanning confocal microscope. (A, D) One-cell embryo $6 \mathrm{~h}$ after fertilization. (B, E) One-cell embryo arrested at the $\mathrm{M}$ phase by nocodazole. (C, F) A PM-embryo. DAPI stainning was used to mark the DNA of the cells (upper panel). 
A

$6 \mathrm{~h}$

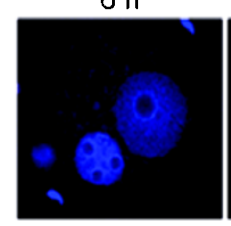

$12 \mathrm{~h}$

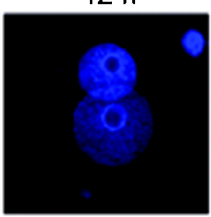

Aphi 6-24 h
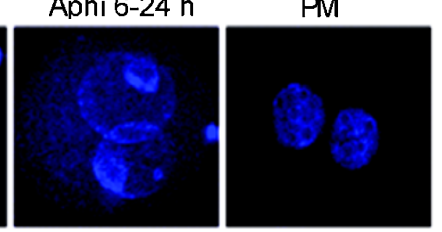

B

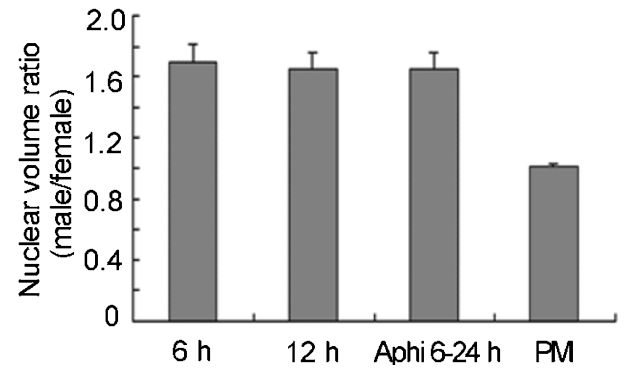

Fig. 4 Relative volume of the nucleus derived from the paternal genome to that derived from the maternal genome. (A) Confocal microscopy images of PM embryos and one-cell embryos at $6 \mathrm{~h}, 12 \mathrm{~h}$ and $24 \mathrm{~h}$ after insemination. The one-cell embryos at $24 \mathrm{~h}$ were treated by aphidicolin from $6 \mathrm{~h}$ to $24 \mathrm{~h}$ after insemination (aphi 6-24 h). (B) Ratio of paternal/maternal nuclear volumes in the embryos described in (A). The numbers of embryos analyzed in $6 \mathrm{~h}, 12 \mathrm{~h}$, aphi 6-24 h, and PM groups were 9, 10, 10, and 23, respectively.

ratio for the two nuclei by dividing the volume of the larger nucleus by that of the smaller one, and found that the ratio was 1.01 . The $\chi^{2}$ fitness test showed that there was no significant difference between the two volumes, suggesting that the nuclei derived from paternal and maternal genomes are approximately equal in volume.

\section{Disappearance of the transcriptional activity asym- metry between the two nuclei in the PM-embryo}

Expression of the parental genomes was detected at the G2 phase in the one-cell stage, demonstrating a significantly higher level of transcription in the male pronucleus than in the female pronucleus (Fig. 5). The level of BrUTP incorporated by the male pronucleus was 2.06-fold higher than that of the female pronucleus. This asymmetric transcriptional activity between parental genomes maintained until $24 \mathrm{~h}$ after insemination, when DNA replication was inhibited by the addition of aphidicolin. In these embryos, the ratio of transcriptional activity between male and female pronucleus was 1.64 . However, the asymmetry was lost in the PM-embryos (Fig. 5). When the nuclei derived from the paternal and maternal genomes were discriminated based on the fluorescence of the anti-methylated H3K9 antibody, no difference in transcriptional activity was detected between the nuclei of different parental origin. The
A

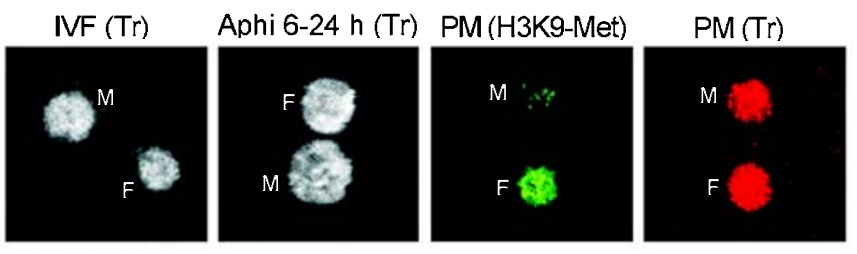

B

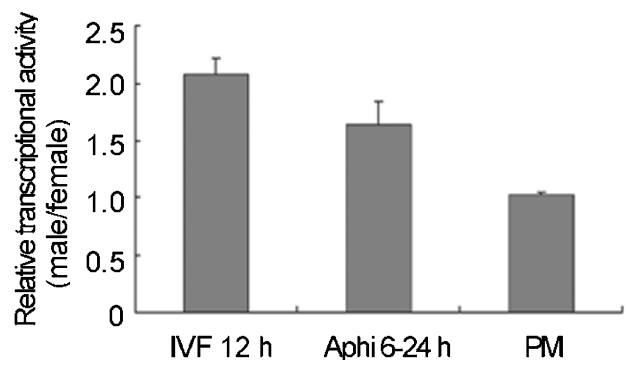

Fig. 5 Relative transcriptional activity of the nucleus derived from the paternal genome to that derived from the maternal genome. (A) Confocal microscopy images of uridine incorporation in the PMembryos and one-cell embryos $12 \mathrm{~h}$ and $24 \mathrm{~h}$ after insemination. The one-cell embryos at $24 \mathrm{~h}$ were treated by aphidicolin from $6 \mathrm{~h}$ to $24 \mathrm{~h}$ after insemination (aphi 6-24 h). M represents the male pronucleus, and $\mathrm{F}$ represents the female pronucleus. Tr, transcriptional activity determined by monitoring the incorporation of BrUTP (red color or shifted to black and white); H3K9-Met, methylation of lysine 9 on histone H3 (green color). (B) Ratio of paternal/maternal transcriptional activity in the embryos described in (A). The numbers of embryos analyzed in the $12 \mathrm{~h}$, aphi 6-24 h, and PM-embryo groups were 26,26 , and 54 , respectively.

ratio of transcriptional activity (paternal/maternal nucleus) was 1.02 , which indicates that the transcriptional activities of the nuclei derived from paternal and maternal genomes are approximately equal in PM-embryos.

\section{DISCUSSION}

In the present study, we developed a new protocol to produce PM-embryos, in which the parental genomes separately formed nuclei in a single karyocyte after exiting from the first mitosis. The one-cell embryos were arrested at metaphase of the first mitosis by treatment with nocodazole, an inhibitor of tubulin polymerization, and were then induced to form nuclei by 6-DMAP, which is a phosphorylation inhibitor [13]. Although nocodazole and 6DMAP have been widely used to inhibit the cell cycle [14, 15], we could not exclude the possibility that the disappearance of the asymmetries on pronuclear volume and transcriptional activity between the male and female genomes was merely caused by the detrimental effects of these two agents. However, it was proved to be unlikely, as one-cell embryos that were treated simultaneously with 
nocodazole and 6-DMAP ( $8 \mathrm{mM})$ at the $\mathrm{G} 2$ stage did not enter metaphase, and they retained the two parental pronuclei with asymmetries of volume and transcriptional activity (data not shown).

Most mitotic events, such as nuclear membrane disintegration, chromosome condensation, and microtubule reorganization, are regulated by reversible phosphorylation of certain proteins $[16,17]$. It was reported that 6 DMAP could prevent germinal vesicle breakdown (GVBD) and chromatin condensation in the GV stage oocytes of mice, cows, and pigs $[18,19]$. Thus, the inhibition of protein phosphorylation reduces the ability of the mouse oocyte to break down the nuclear envelope and condense the chromosomes. Although phosphorylation-dependent disassembly of the nuclear membrane and chromosomal condensation has been well characterized during cell cycle progression through the $\mathrm{G} 2$ to $\mathrm{M}$ phases, it is not known whether the dephosphorylation events are sufficient to induce nuclear envelope formation and chromosomal decondensation during the $\mathrm{M}$ to $\mathrm{G} 1$ phases. The results of the present experiments suggest that inhibition of protein phosphorylation by 6-DMAP could induce the formation of the nuclear envelope and chromosomal decondensation in the cells, which are arrested at metaphase. Therefore, this protocol may prevent the loss of chromosomes in somatic cell hybrids, in which the two nuclei of the heterokaryon (a single cell that contains two different nuclei in a common cytoplasm) fuse and frequently lose chromosomes in culture. In addition, this study provides a model to investigate the regulation and function of specific transacting factors concerning gene expression in eukaryotes.

It is generally accepted that maturation promoting factor (MPF) is responsible for the induction of nuclear envelope breakdown (NEBD) and chromatin condensation. In all species studied, the appearance of MPF has been associated with a high level of protein phosphorylation [20]. The activity of MPF fluctuate concordantly with protein phosphorylation. The induction of nuclear envelope formation and chromosome decondensation by 6-DMAP may lie in the following mechanism: 6-DMAP induces the dephosphorylation of MPF and its relevant proteins or inhibits their phosphorylation, and in turn the decreased activity of MPF induces the formation of nuclei and the decondensation of chromosomes in embryos that were arrested at metaphase.

During the production of cloned animals, previous studies suggest that the exposure of somatic nuclei to metaphase cytoplasm is an important process to ensure successful reprogramming of the genome and support for embryonic development [21-24]. It seems likely that NEBD and chromosomal condensation of the donor nuclei are both critical processes in genome reprogramming after trans- fer into recipient oocytes. In contrast, when activated oocytes with reduced MPF activity were used as recipients, the membranes and chromosomes of the transferred somatic nuclei remained intact and decondensed, respectively, and nuclear remodeling was unsuccessful in these reconstructed oocytes [24]. These results suggest that direct interaction of the chromosome with the cytoplasmic environment is necessary for chromosome remodeling. And NEBD may play an important role in the reprogramming of differentiated nuclei by allowing direct interactions of chromosomes with cytoplasmic factor(s) in the oocytes. In the present experiments, the asymmetries of the nuclear volumes and transcriptional activities between parental genomes disappeared after the chromosomes interacted directly with the cytoplasm (Figs. 4 and 5). At the M phase, cytoplasmic factors are allowed to gain access to the chromosomes by NEBD, resulting in the reprogramming of the parental genomes. Our results suggest that the first mitosis presents a window for resetting the different transcriptional programs of parental genomes so that they are equivalent to each other.

Although many of the differences between male and female genomes are erased during the first cell cycle, asymmetric histone $\mathrm{H} 3$ methylation at lysine 9 (H3/K9) persists. In mouse embryos, methylated histone $\mathrm{H} 3 / \mathrm{K} 9$ is restricted to maternal but not paternal chromatin before the four-cell stage [10-12]. In the current study, the asymmetric H3K9 methylation in the parental genomes was retained in the PM-embryos (Fig. 3), although the maternal and paternal genomes showed the same levels of transcriptional activity (Fig. 5). Recent findings suggest that $\mathrm{H} 3 / \mathrm{K} 9$ methylation is involved in the formation of the constitutive heterochromatin as well as the facultative heterochromatin of the inactive X chromosome [25-29]. H3/K9 methylation has also been associated with the silencing of euchromatic genes [29-31]. The biological significance of the maintenance of asymmetric $\mathrm{H} 3 / \mathrm{K} 9$ methylation during early development still remains to be resolved.

Received, Apr 10, 2004

Revised, Nov 17, 2004

Accepted, Dec 11, 2004

\section{REFERENCES}

1 Aoki F, Worrad DM, Schultz RM. Regulation of transcriptional activity during the first and second cell cycles in the preimplantation mouse embryo. Dev Biol 1997; 181:296-307.

2 Ram PT, Schultz RM. Reporter gene expression in G2 of the 1cell mouse embryo. Dev Biol 1993; 156:552-6.

3 Bouniol C, Nguyen E, Debey P. Endogenous transcription occurs at the 1-cell stage in the mouse embryo. Exp Cell Res 1995; 218:57-62.

4 Adenot PG., Mercier Y, Renard JP, Thompson EM. Differential 
H4 acetylation of paternal and maternal chromatin precedes DNA replication and differential transcriptional activity in pronuclei of 1-cell mouse embryos. Development 1997; 124:4615-25.

5 Mayer W, Niveleau A, Walter J, Fundele R, Haaf T. Demethylation of the zygotic paternal genome. Nature 2000; 403:501-3.

6 Cho T, Sakai S, Nagata M, Aoki F. Involvement of chromatin structure in the regulation of mouse zygotic gene activation. Animal Science Journal 2002; 73:113-22.

7 Martinez-Balbas MA, Dey A, Rabindran SK, Ozato K, Wu C. Displacement of sequence-specific transcription factors from mitotic chromatin. Cell 1995; 83:29-38.

8 Whitten, WK. Nutrient requirement for the culture of preimplantation embryos. Adv Biosci 1971; 6:129-39.

9 Chatot CL, Ziomek CA, Bavister BD, Lewis JL, Torres I. An improved culture medium supports development of randombred 1-cell mouse embryos in vitro. J Reprod Fertil 1989; 86: 679-88.

10 Arney KL, Bao S, Bannister AJ, Kouzarides T, Surani MA. Histone methylation defines epigenetic asymmetry in the mouse zygote. Int J Dev Biol 2002; 46:317-20.

11 Cowell IG, Aucott R, Mahadevaiah SK, et al. Heterochromatin, HP1 and methylation at lysine 9 of histone H3 in animals. Chromosoma 2002; 111:22-36.

12 Liu H, Kim JM, Aoki F. Regulation of histone H3 lysine 9 methylation in oocytes and early preimplantation embryos. Development 2004; 131:2269-80.

13 Neant I, Guerrier P. 6-Dimethylaminopurine blocks starfish oocyte maturation by inhibiting a relevant protein kinase activity. Exp Cell Res 1988; 176:68-79.

14 Anderiesz C, Fong CY, Bongso A, Trounson AO. Regulation of human and mouse oocyte maturation in vitro with 6-dimethylaminopurine. Hum Reprod 2000; 15:379-88.

15 Samake S, Smith LC. Effects of cell-cycle arrest agents on cleavage and development of mouse embryos. J Exp Zool 1996; 274: 111-20.

16 Morgan DO, Kaplan JM, Bishop JM, Varmus HE. Mitosisspecific phosphorylation of $\mathrm{p} 60^{\mathrm{c}-\mathrm{src}}$ by $\mathrm{p} 34^{\mathrm{cdc} 2}$ associated protein kinase. Cell 1989; 57:775-86.

17 Otto H, Dreger M, Bengtsson LK, Hucho F. Identification of tyrosine-phosphorylated proteins associated with the nuclear envelope. Eur J Biochem 2001; 268:420-8.

18 Motlik J, Rimkevicova Z. Combined effect of protein phosphorylation and protein symthesis inhibitors on maturation of mouse oocytes in vitro. Mol Reprod Dev 1990; 27:230-4.

19 Fulka JJr, Leibfried-Rutledge ML, First NL. Effect of 6-dimethylaminopurine (6-DMAP) on germinal vesicle breakdown (GVBD) of bovine oocytes. Mol Reprod Dev 1991; 29:379-84.

20 Neant I, Guerrier P. Meiosis reinitiation in the molusc Patella vulgata. Regulation of MPF, CSF and chromosome condensation activity by intracellular $\mathrm{pH}$, protein synthesis, and phosphorylation. Development 1988; 102:505-16.

21 Diberardino MA, Hoffner NJ, Orr NH. Genomic potential of erythroid and leukocytic cells of Rana pipiens analyzed by nuclear transfer into diplotene and maturing oocytes. Differentiation 1992; 50:1-13.

22 Wakayama T, Perry ACF, Zuccotti M, Johnson KR, Yanagimachi R. Full-term development of mice from enucleated oocytes injected with cumulus cell nuclei. Nature 1998; 394:369-72.

23 Dominko T, Mitalipova M, Haley B, et al. Bovine oocyte cytoplasm supports development of embryos produced by nuclear transfer of somatic cell nuclei from various mammalian species. Biol Reprod 1999; 60:1496-502.

24 Kim JM, Ogura A, Nagata M, Aoki F. Analysis of the mechanism for chromatin remodeling in embryos reconstructed by somatic nuclear transfer. Biol Reprod 2002; 67:760-6.

25 Heard E, Rougeulle C, Arnaud D, et al. Methylation of histone $\mathrm{H} 3$ at lys-9 is an early mark on the X chromosome during X inactivation. Cell 2001; 107:727-38.

26 Jacobs SA, Tavema SD, Zhang Y, et al. Specificity of the HP1 chromo domain for the methylated N-terminus of histone H3. EMBO J 2001; 20:5232-41.

27 Noma K, Allis CD, Grewal SI. Transitions in distinct histone H3 methylation patterns at the he terochromatin domain boundaries. Science 2001; 293:1150-5.

28 Boggs BA, Cheung P, Heard E, Spector DL, Chinault AC, Allis CD. Differentially methylated forms of histone $\mathrm{H} 3$ show unique association patterns with inactive human X chromosomes. Nat Genet 2001; 30:73-6.

29 Peters AH, Mermoud JE, O'Carroll D, et al. Histone H3 lysine 9 methylation is an epigenetic imprint of facultative heterochromatin. Nat Genet 2002; 30:77-80.

30 Hwang KK, Eissenberg JC, Worman HJ. Transcriptional repression of euchromatic genes by Drosophila heterochromatin protein 1 and histone modifiers. Proc Natl Acad Sci USA 2001; 98:11423-7.

31 Nielsen SJ, Schneider R, Bauer UM, et al. Rb targets histone H3 methylation and HP1 to promoters. Nature 2001; 412:561-5. 